\title{
Insights into Drug Precipitation Kinetics during In Vitro Digestion of a Lipid-Based Drug Delivery System Using In-Line Raman Spectroscopy and Mathematical Modeling
}

\author{
Cordula Stillhart • Georgios Imanidis • Martin Kuentz
}

Received: 9 November 2012 / Accepted: 28 January 2013 / Published online: 28 February 2013

(C) Springer Science+Business Media New York 2013

\begin{abstract}
Purpose To determine drug precipitation during in vitro lipolysis of a lipid-based drug delivery system (LBDDS) using Raman spectroscopy as a real-time monitoring technique. A second aim was to describe the kinetics of lipolysis-triggered drug precipitation using a theoretical nucleation and growth model. Methods A model LBDDS containing different concentration of fenofibrate was digested in vitro and drug precipitation was determined after ultracentrifugation and nanofiltration (off-line methods), as well as by Raman spectroscopy (in-line method). Subsequently, a theoretical nucleation and growth model was fitted to the obtained drug crystallization profiles by considering the lipolysis-triggered change in drug solubility.

Results Compared with standard off-line measurements, Raman spectroscopy enabled a more robust and highly timeresolved analysis of lipolysis-triggered drug precipitation. Although the formulation was rapidly digested, fenofibrate remained in a supersaturated state for several minutes before beginning to crystallize. The in vitro digestion results were in excellent agreement with the theoretical model $\left(R^{2}>0.976\right)$.

Conclusions The combination of real-time Raman spectroscopy and mathematical modeling provided insights into the kinetics of lipolysis-triggered drug crystallization. This knowledge allows a better biopharmaceutical understanding and will, ultimately, lead to the improved development of lipid-based drug formulations.
\end{abstract}

\footnotetext{
C. Stillhart $\cdot$ G. Imanidis $\cdot$ M. Kuentz $(\square)$

University of Applied Sciences and Arts Northwestern Switzerland School of Life Sciences, Institute of Pharma Technology

Gründenstrasse 40

4132 Muttenz, Switzerland

e-mail: martin.kuentz@fhnw.ch

C. Stillhart $\cdot$ G. Imanidis

University of Basel, Department of Pharmaceutical Sciences

Klingelbergstrasse 50

4056 Basel, Switzerland
}

$\begin{array}{ll}\text { KEY WORDS } \text { in vitro lipolysis · kinetics of precipitation } \\ \text { lipid-based drug delivery systems · Raman spectroscopy } \\ \text { supersaturation } \\ \text { ABBREVIATIONS } \\ \text { 4-BPBA } & \text { 4-bromophenylboronic acid } \\ \text { DG } & \text { Diglycerides } \\ \text { DLS } & \text { Dynamic light scattering } \\ \text { FA } & \text { Fatty acids } \\ \text { HPLC } & \text { High-performance liquid chromatography } \\ \text { LBDDS } & \text { Lipid-based drug delivery systems } \\ \text { MG } & \text { Monoglycerides } \\ \text { MSC } & \text { Multiple scattering correction } \\ \text { NaTDC } & \text { Sodium taurodeoxycholate } \\ \text { PC } & \text { Phosphatidylcholine } \\ \text { PDI } & \text { Polydispersity index } \\ \text { PLS } & \text { Partial least squares } \\ \text { RMSEC } & \text { Root-mean-square error of calibration } \\ \text { RMSECV } & \text { Root-mean-square error of cross-validation } \\ \text { SG } & \text { Savitzky-Golay } \\ \text { S(M)EDDS } & \text { Self-(micro)emulsifying drug delivery system } \\ \text { SNV } & \text { Standard normal variate } \\ \text { TG } & \text { Triglycerides } \\ \text { XRPD } & \text { X-ray powder diffraction } \\ \end{array}$

\section{INTRODUCTION}

Contemporary drug discovery screening has generated an increasing number of highly active but poorly water-soluble drug candidates. Lipid-based drug delivery systems (LBDDS) are an innovative strategy that significantly enhances the oral bioavailability of such compounds. Because the formulated drug is already in a dissolved state, the critical dissolution step is circumvented. This is, however, only one mechanism by which LBDDS can enhance oral drug absorption. Drug solubilization in the intestinal medium is generally improved in the presence of lipidic excipients. Furthermore, these excipients can stabilize 
a supersaturated drug, may reduce pre-systemic clearance, and can promote lymphatic absorption (1-4).

The improvement of oral bioavailability is largely governed by the fate of the LBDDS in the gut. In addition to formulation dispersion, digestion represents a particularly critical step, because hydrolyzed glycerides and some surfactants exhibit a change in polarity (5-7). Thus, the capacity of a formulation to solubilize a drug may be progressively reduced, developing an increased risk of drug precipitation. To predict the fate of a drug in the gastrointestinal lumen therefore requires adequate in vitro tests. Although no standard compendial methods have been established so far, first attempts have been made in this direction (7-10). Most recently, the Lipid Formulation Classification System Consortium released a collaborative work, which was aimed at standardizing in vitro methods to assess the performance of LBDDS, with a particular focus on lipolysis testing $(11,12)$.

A lipolysis test should primarily reveal whether a formulation keeps the drug solubilized. According to the current procedure, samples are removed at given time intervals during a digestion experiment $(11,13)$. After enzyme inhibition, the digests are separated by ultracentrifugation into a pellet phase (containing precipitated drug), an oil phase, and an aqueous phase. The drug distribution in these phases is crucial, because the solubilized drug is expected to approximate the dose fraction that is available for absorption (14).

The influence of lipid digestion on drug solubilization is the result of a complex interplay between several factors. Relevant for solubilization are the properties of the drug and of the lipolysis products in the presence of bile salts and phospholipids. It is assumed that colloidal structures, which are generated during formulation lipolysis, largely define drug solubilization. Although these structures have been characterized in several studies (15-21), it is difficult to predict the resulting drug solubilization. The difficulty arises not only from dynamic changes in the medium but also from the possibility of drug supersaturation. Indeed, if the period of supersaturation is long enough to enable drug absorption, then intestinal drug precipitation might be prevented. Thus, considerable research efforts have focused on drug supersaturation in biorelevant media, with and without the aid of lipid-based excipients $(4,22,23)$. As well as drug supersaturation, the solid-state properties of precipitated drug are of biopharmaceutical relevance. Sassene et al. recently observed the precipitation of amorphous cinnarizine during digestion of a self-emulsifying system (24). Drug redissolution was measured in vitro and proved to be faster than from its crystalline form. Such knowledge appears to be critical to the development of a formulation and indicates that more research is needed in this pharmaceutical field.

Formulation digestion and drug solubilization are highly dynamic processes, but the lack of real-time information limits the feasibility of exploring in vitro digestion. Warren et al. recently demonstrated the importance of using an in situ method (synchrotron small-angle X-ray scattering) to gain insight into a rapidly proceeding digestion process (25). Significant transformations of the formulation typically occur within a short time frame and the sampling regime of traditional methods almost precludes the detection of transient solubility effects.

The use of Raman spectroscopy is an efficient approach to the real-time monitoring of crystallization processes during chemical production (26-31). The method allows rapid spectra collection in a contact-free manner and with high chemical specificity. Recently, Raman spectroscopy has been applied successfully to study drug precipitation in a biorelevant medium (32) and for process monitoring in complex multiphasic systems (33). These are promising features with respect to real-time monitoring of drug crystallization during in vitro digestion. However, the complexity of the medium and the varying composition during lipolysis are particularly challenging for any spectroscopic application.

The first aim of the study was to evaluate the potential of Raman spectroscopy for real-time monitoring of drug precipitation during in vitro lipolysis. Based on this in situ information, the second aim was to describe the kinetics of drug precipitation using a theoretical model of nucleation and particle growth, including the lipolysis-triggered change in drug solubility. Finally, using the combination of real-time spectroscopy and mathematical modeling, we aimed to gain a better quantitative understanding of lipolysis-triggered drug precipitation.

\section{MATERIALS AND METHODS}

\section{Materials}

We obtained fenofibrate (2-[4-(4-chlorobenzoyl)phenoxy]2-methylpropionic acid 1-methylethyl ester, $\geq 99 \%$ ), Trizma ${ }^{\circledR}$ maleate, calcium chloride dihydrate $(\geq 99 \%)$, pancreatin (from porcine pancreas, 8xUSP specifications), 4bromophenylboronic acid (4-BPBA, $\geq 95.0 \%$ ), chloroform, and acetonitrile from Sigma-Aldrich Chemie $\mathrm{GmbH}$ (Buchs, Switzerland), and sodium chloride ( $\geq 99 \%$ ) from Carl Roth GmbH (Karlsruhe, Germany). Lipoid E PC S (phosphatidylcholine from egg yolk) was supplied by Lipoid GmbH (Ludwigshafen, Germany), sodium taurodeoxycholate by Prodotti Chimici e Alimentari S.p.A. (Basaluzzo, 
Italy), and sodium hydroxide $1 \mathrm{M}$ by Scharlab S.L. (Sentmenat, Spain). Imwitor ${ }^{\circledR} 988$ was purchased from Sasol Germany GmbH (Witten, Germany), Miglyol 812 N from Hänseler AG (Herisau, Switzerland), and Cremophor ${ }^{\circledR}$ RH 40 from BASF AG (Ludwigshafen, Germany). Imwitor ${ }^{\circledR} 988$ was a blend of medium-chain mono-, di-, and triglycerides $(54.6 \%$ monoglyceride [MG], $38.0 \%$ diglyceride [DG], and $7.1 \%$ triglyceride [TG]) consisting of $98.7 \% w / w$ caprylic acid (C8), $1.1 \%$ w/w capric acid (C10), and $0.1 \%$ w/w caproic acid (C6), with average molecular weights of 197, 340, and $483 \mathrm{~g} / \mathrm{mol}$ for MG, DG, and TG, respectively (according to the certificate of analysis, lot no. 003041, Cremer Oleo GmbH). Miglyol $812 \mathrm{~N}$ was a medium-chain TG consisting of $57.9 \% w / w$ caprylic acid (C8), 41.2\% w/w capric acid (C10), $0.5 \%$ w/w lauric acid (C12), and $0.1 \% w / w$ caproic acid (C6) with an average molecular weight of $517 \mathrm{~g} / \mathrm{mol}$ (according to the certificate of analysis, lot no. 2008111435, Hänseler AG).

Purified water was prepared with an Arium ${ }^{\circledR} 61215$ water-purification system from Sartorius Stedim Biotech GmbH (Göttingen, Germany). We used Anotop ${ }^{\circledR} 25$ Plus filters (aluminum oxide, $0.1 \mu \mathrm{m}$ ) purchased from Whatman GmbH (Dassel, Germany) for nanofiltration.

\section{Preparation of the Model Formulation}

A self-microemulsifying drug delivery system (SMEDDS) was selected as the model formulation. The SMEDDS was composed of $40 \%$ w/w Miglyol 812, 20\% w/w Imwitor ${ }^{\circledR} 988$, and $40 \%$ w/w Cremophor ${ }^{\circledR}$ RH 40 . The components were mixed on a magnetic stirrer at $40^{\circ} \mathrm{C}$ until a clear solution was obtained and then slowly cooled to room temperature.

Fenofibrate $(\log P 4.6)$ was used as a poorly water-soluble model drug. The compound was incorporated into the formulation at levels of $20,30,40,50,60,70$, and $80 \mathrm{mg} / \mathrm{g}$ $\left(C_{\text {form }}\right)$. All formulations were visually assessed for absence of undissolved drug particles.

\section{In Vitro Lipolysis Test}

The in vitro lipolysis test was performed as described in the literature (7). We prepared a micellar solution containing $1.25 \mathrm{mM}$ phosphatidylcholine (PC) and $5 \mathrm{mM}$ sodium taurodeoxycholate (NaTDC) to simulate fasted state intestinal conditions. PC was dissolved in chloroform and the solvent was evaporated under vacuum (Rotavapor RE 120, Büchi, Switzerland). NaTDC and digestion buffer $(50 \mathrm{mM}$ Trizma ${ }^{\circledR}$ maleate, $150 \mathrm{mM} \mathrm{NaCl}$, and $5 \mathrm{mM}$ $\mathrm{CaCl}_{2} \cdot 2 \mathrm{H}_{2} \mathrm{O}$; pH7.5) were then added and the mixture was stirred for $12 \mathrm{~h}\left(450 \mathrm{rpm}, 5^{\circ} \mathrm{C}\right)$. To prepare the pancreatin extract, we mixed $1 \mathrm{~g}$ of porcine pancreatin powder per $5 \mathrm{ml}$ of digestion buffer $\left(5^{\circ} \mathrm{C}\right)$, stirred for $15 \mathrm{~min}$, and then centrifuged the suspension $\left(15 \mathrm{~min}, 1,600 \mathrm{xg}, 5^{\circ} \mathrm{C}\right)$. Finally, the supernatant was collected and the $\mathrm{pH}$ adjusted to 7.5 , which corresponded to the $\mathrm{pH}$ of the lipolysis medium. The pancreatin extract was freshly prepared each day and stored on ice until use.

The micellar solution $(108 \mathrm{ml})$ was transferred to a thermostated glass vessel $\left(37.0 \pm 0.5^{\circ} \mathrm{C}\right)$ and the formulation $(2.6 \mathrm{~g})$ was added. The mixture was stirred for $10 \mathrm{~min}$ for complete dispersion, thermal equilibration, and $\mathrm{pH}$ adjustment to $7.500 \pm 0.001$. For mixing, we used a magnetic stirrer $(3 \mathrm{~cm}$ in diameter) at a speed of $450 \mathrm{rpm}$. Digestion was initiated by the addition of $12 \mathrm{ml}$ pancreatin extract (final nominal lipase activity: 1000 tributyrin units per $\mathrm{ml}$ ). The free fatty acids (FA) produced during lipolysis were titrated using $1 \mathrm{M} \mathrm{NaOH}$ to maintain $\mathrm{pH} 7.500$ using a $\mathrm{pH}$-stat apparatus (842 Titrando and 800 Dosino, Metrohm AG, Switzerland), which was operated using the Tiamo 1.2 software package (Metrohm AG, Switzerland). Lipolysis was allowed to proceed for $30 \mathrm{~min}$.

To determine the $\mathrm{NaOH}$ consumption caused by digestion of the blank digestion medium, we performed the lipolysis test with pure digestion medium without formulation $(n=3)$.

Fenofibrate formulations with varying drug loads (20, 30, $40,50,60,70$, and $80 \mathrm{mg} / \mathrm{g}$ ) were digested in triplicate and the amount of precipitated and/or solubilized drug was assayed using three different procedures, i.e., ultracentrifugation, nanofiltration, and Raman spectroscopy.

\section{"Back-Titration" and Calculation of Free Fatty Acids Liberated During Digestion}

It was previously shown that the titrated $\mathrm{NaOH}$ volume represents only an approximation of the free fatty acids (FA) liberated during lipolysis. Some FA exist in their unionized state, according to the $\mathrm{pK}_{\mathrm{a}}$ value, leading to an underestimation of the total FA liberation. This must be corrected by a procedure that was previously termed "back-titration" $(34,35)$. We performed the back-titration experiment as described in the literature, with minor changes. At the end of the 30 min digestion period, the $\mathrm{pH}$ was rapidly increased to 9 by addition of $1 \mathrm{M} \mathrm{NaOH}$ for complete FA ionization (deprotonation). The experiment was also done with blank digestion buffer including pancreatin extract (without formulation, $\mathrm{PC}$, and NaTDC) to determine the $\mathrm{NaOH}$ volume needed to increase the $\mathrm{pH}$ in the absence of lipolysis products. The latter volume was subtracted from the total back-titration volume obtained in the presence of lipolysis products to obtain 
the value of $F A_{\text {titr }}$ (back-titration). The correction factor was calculated according to the formula:

Correction factor $=\frac{F A_{\text {titr }}(\text { direct titration })+F A_{\text {titr }}(\text { back }- \text { titration })}{F A_{\text {titr }}(\text { direct titration })}$

where $F A_{\text {titr }}$ (direct titration) is the amount of FA titrated after the $30 \mathrm{~min}$ digestion period.

A correction factor was calculated for both the lipolysis of formulations and the lipolysis of pure digestion medium (without formulation). Finally, the amount of FA liberated from the pure digestion medium was subtracted from the total amount of FA titrated in presence of the SMEDDS.

\section{Determination of Drug Precipitation}

\section{Ultracentrifugation Method}

During formulation lipolysis, aliquots $(2.7 \mathrm{ml})$ were taken from the digestion media after a 1, 2.5, 5, 7.5, 10, 12.5, 15, 20,25 , and $30 \mathrm{~min}$ digestion period. We immediately added $20 \mu \mathrm{l}$ of a 4-BPBA solution (1 M in methanol) to inhibit further lipolysis (36) and ultracentrifuged the samples $\left(80,000 \times g, 37^{\circ} \mathrm{C}, 90 \mathrm{~min}\right)$ in a Centrikon T-1 180 ultracentrifuge equipped with a TFT-80.4 fixed-angle rotor (Kontron Instruments, Milan, Italy). The aqueous phase was then diluted with acetonitrile and centrifuged (16,000xg, $15 \mathrm{~min})$. The pellet was suspended in purified water, diluted in acetonitrile, and centrifuged $(16,000 \mathrm{xg}, 15 \mathrm{~min})$. Finally, all samples were analyzed using high-performance liquid chromatography (HPLC).

\section{Nanofiltration Method}

A $2 \mathrm{ml}$ sample was withdrawn from the lipolysis medium after $1,2.5,5,7.5,10,12.5,15,20,25$, and $30 \mathrm{~min}$. The samples were directly passed through an Anotop ${ }^{\circledR} 25$ Plus syringe filter (pore size $0.1 \mu \mathrm{m}$, aluminum oxide filter membrane) (37) and the filtrate was immediately diluted in acetonitrile to stop further lipolysis. The samples were then centrifuged $(16,000 \mathrm{xg}, 30 \mathrm{~min})$ and analyzed by HPLC. We verified experimentally that no loss of dissolved substance occurred through adsorption onto the filter material.

The Mann-Whitney test was performed to compare the concentration of solubilized drug after 0 and $1 \mathrm{~min}$ of lipolysis. For statistical treatments we used the software Statgraphics ${ }^{\circledR}$ Centurion XV Professional (Statpoint Technologies Inc. Warrenton, USA) and selected a significance level of $95.0 \% \quad(p<0.05)$.
In-line Determination of Drug Precipitation Using Raman Spectroscopy

A multi-fiber Raman $\mathrm{P}^{h} \mathrm{AT}$ probe was used to determine the course of fenofibrate precipitation in situ. The titration vessel was covered with a custom-built vessel lid (Metrohm AG, Zofingen, Switzerland) with an extra cavity for the $\mathrm{P}^{h} \mathrm{AT}$ probe, which was positioned $1 \mathrm{~cm}$ above the initial level of the lipolysis medium (Fig. 1). A comparatively large titration vessel, and thus a relatively high volume of digestion medium, were required because of the $\mathrm{P}^{h} \mathrm{AT}$ probe size (outer diameter: $32 \mathrm{~mm}$ ). The entire lipolysis vessel was wrapped in aluminum foil and the laboratory was darkened to avoid the influence of external light on the Raman signal.

We used a Raman RXN1 analyzer (Kaiser Optical Systems, Inc., Ann Arbor, MI, USA) equipped with a charge-coupled device (CCD) camera and a diode laser operating at a wavelength of $785 \mathrm{~nm}$. The spectra were acquired with a laser power of $400 \mathrm{~mW}$ and background Rayleigh scattering was removed by a holographic filter during spectra acquisition. A single spectrum was acquired every $20 \mathrm{~s}$ (acquisition time $18.5 \mathrm{~s}$ ) with a resolution of $4 \mathrm{~cm}^{-1}$ using the $\mathrm{iC}$ Raman Instrument software (Version 3.0, Mettler-Toledo AutoChem Inc., Columbia, MD, USA). The Raman $\mathrm{P}^{h} \mathrm{AT}$ probe was a multi-fiber probe with a non-contact sampling optic device having a laser spot diameter of $6 \mathrm{~mm}$. The scattered radiation was collected by an array of 50 optical fibers and delivered to the CCD camera.

To remove sources of non-linearity and spectral information that was uncorrelated with the concentration of the analyte, the spectra were subjected to several preprocessing algorithms, i.e., the multiplicative scatter correction (MSC) (38), the standard normal variate (SNV)

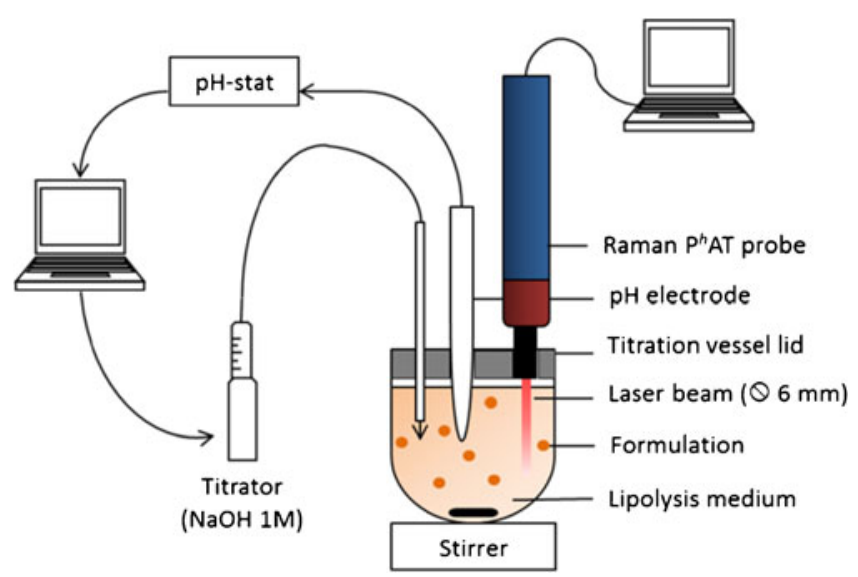

Fig. I Experimental setup of the in vitro lipolysis test combined with Raman spectroscopy. The $\mathrm{P}^{h} \mathrm{AT}$ probe consisted of a non-contact optic device and provided a large laser spot area (6 $\mathrm{mm}$ in diameter). 
transformation (39), and the Savitzky-Golay (SG) polynomial derivative filter (40). All data were mean-centered and, finally, a calibration model was built by partial least-squares (PLS) regression (41). The optimal number of PLS factors was defined where the root-mean-square error of crossvalidation (RMSECV) reached a minimum $(<1 \%$ of spectral variation). Cross-validation was performed with the leave-one-out method. We evaluated different spectral ranges to include in the PLS regression model, i.e., the entire spectrum $\left(500-1800 \mathrm{~cm}^{-1}\right)$, the fingerprint range of fenofibrate $\left(1000-1800 \mathrm{~cm}^{-1}\right)$, and specific fenofibrate peaks $\left(1132-1164 \mathrm{~cm}^{-1}, 1550-1615 \mathrm{~cm}^{-1}\right.$, and 1635$\left.1663 \mathrm{~cm}^{-1}\right)$. Spectral preprocessing and PLS regression analysis were calculated using MATLAB ${ }^{\circledR}$ (MathWorks, Naticks, MA, USA). The calibration models were evaluated in terms of the correlation coefficient $R^{2}$, the crossvalidation coefficient $Q^{2}$, the root-mean-square error of calibration (RMSEG), and the RMSECV.

\section{Drug Solubilization Upon Dispersion Without Lipolysis}

Drug solubilization under non-digesting conditions was determined to assess whether fenofibrate precipitated spontaneously upon aqueous dispersion of the formulation (without lipolysis). Thus, $2.6 \mathrm{~g}$ of SMEDDS with $80 \mathrm{mg} / \mathrm{g}$ fenofibrate were dispersed in $108 \mathrm{ml}$ of digestion buffer (containing NaTDC and $\mathrm{PC}$ ) in a glass vessel at $37^{\circ} \mathrm{C}$ and stirred at $450 \mathrm{rpm}$. After equilibrating the system for $10 \mathrm{~min}, 12 \mathrm{ml}$ of pure digestion buffer (without pancreatin extract) were added and the medium was stirred for $30 \mathrm{~min}$. Subsequently, three $2 \mathrm{ml}$ aliquots were centrifuged for $30 \mathrm{~min}\left(16,000 \mathrm{xg}, 37^{\circ} \mathrm{C}\right)$ and the concentration of solubilized and precipitated fenofibrate was measured by HPLC. The experiment was carried out in triplicate.

\section{Determination of Drug Solubility}

Drug solubility was determined in the undiluted formulation $\left(C_{\text {form }}^{*}\right)$. Excess solid drug was added to a $2 \mathrm{ml}$ aliquot of blank formulation and stored in hermetically sealed glass vials during equilibration $\left(37^{\circ} \mathrm{C}, 450 \mathrm{rpm}\right)$. After $24 \mathrm{~h}, 48 \mathrm{~h}$, and $72 \mathrm{~h}$ the samples were centrifuged $\left(37^{\circ} \mathrm{C}, 16,000 \mathrm{xg}\right.$, $30 \mathrm{~min}$ ) and the supernatant was analyzed by HPLC. Equilibrium was assumed when two consecutive solubility samples varied by $\leq 5 \%(w / w)$.

Furthermore, we determined the drug solubility $\left(C_{m, t}^{*}\right)$ in the lipolysis medium containing drug-free SMEDDS after a $1,2.5,5,7.5,10,12.5,15,20,25$, and 30 min digestion period. The enzyme inhibitor (4-BPBA, $1 \mathrm{M}$ in methanol,
$9 \mu \mathrm{l}$ per $\mathrm{ml}$ of digest) was added to the medium at the aforementioned time points to stop further digestion. Excess solid drug was transferred to a $10 \mathrm{ml}$ aliquot of the lipolysis medium and was hermetically sealed in glass vials. After equilibration $\left(450 \mathrm{rpm}, 37^{\circ} \mathrm{C}\right)$, the samples were ultracentrifuged $\left(80,000 \times \mathrm{g}, 37^{\circ} \mathrm{C}, 90 \mathrm{~min}\right)$. The liquid phase was then diluted in acetonitrile, centrifuged $(16,000 \mathrm{xg}, 15 \mathrm{~min})$, and finally analyzed by HPLC. Because the solubilities were found to decrease at equilibration times longer than $24 \mathrm{~h}$, we considered the value obtained at $24 \mathrm{~h}$ of equilibration as the relevant solubility. The observed decrease in solubility was attributed to kinetic instability of the colloidal structures in digested medium $(4,12)$. Whenever a lipid phase was obtained upon ultracentrifugation, the lipid and the aqueous phase were re-emulsified, to measure the overall concentration of solubilized drug. The drug solubility experiments were carried out in triplicate.

\section{High-Performance Liquid Chromatography (HPLC)}

For HPLC analysis (Agilent Technologies 1200 Series) we used an isocratic pump (G1310A), an autosampler (G1329A), and a variable wavelength detector $(\mathrm{G} 1310 \mathrm{~A})$. All measurements were done on a LiChrospher ${ }^{\circledR}$ 60, RP select B 125-4 (5 $\left.\mu \mathrm{m}\right)$ column (Merck, Darmstadt, Germany), using a flow rate of $1 \mathrm{ml} / \mathrm{min}$, a UV detection wavelength of $287 \mathrm{~nm}$, and an injection volume of $20 \mu \mathrm{l}$. The mobile phase consisted of acetonitrile and ammonium acetate buffer (pH3.5; $25 \mathrm{mM})$ at a ratio of 65:35 $(v / v)(42)$. All sample measurements were in the linear range of calibration.

\section{Dynamic Light Scattering (DLS)}

We measured the particle size of the dispersed formulation before lipolysis initiation by DLS using a Zeta Sizer Nano ZS (Malvern Instruments, Malvern, UK), equipped with a $4 \mathrm{~mW}$ He-Ne Laser operating at a wavelength of $633 \mathrm{~nm}$. The scattering signal was detected at an angle of $173^{\circ}$ and each sample was measured in triplicate for $10 \mathrm{~min}$. The result was expressed as intensity averaged particle diameter $(\mathrm{nm})$ and as polydispersity index (PDI).

\section{X-ray Powder Diffraction (XRPD)}

The pellet phase that was obtained upon digestion and ultracentrifugation of the drug-containing formulation was analyzed by XRPD. The formulation with $80 \mathrm{mg} / \mathrm{g}$ fenofibrate was digested for $30 \mathrm{~min}$ in the in vitro lipolysis test and, after enzyme inhibition, an aliquot was ultracentrifuged $\left(80,000 \times g, 37^{\circ} \mathrm{C}, 90 \mathrm{~min}\right)$. The pellet phase was 
immediately isolated and an X-ray diffractogram was recorded. The same procedure was followed with the pellet phase obtained from a $30 \mathrm{~min}$ lipolysis experiment with drug-free formulation, which was spiked with an equal amount of pure fenofibrate (as used for formulation preparation). Moreover, we recorded the X-ray diffractogram of crystalline fenofibrate as a reference.

We used a theta-theta X-ray powder diffractometer (R-XRD Phaser D2, Bruker AXS GmbH, Karlsruhe, Germany) equipped with a Co and $\mathrm{Cu}$ KFL tube $(30 \mathrm{kV}$, $10 \mathrm{~mA})$ as radiation source and a Lynxeye ${ }^{\circledR}$ detector. The samples were scanned in the angular range of $5^{\circ}(2 \theta)$ to $40^{\circ}(2 \theta)$ with a step size of $0.1^{\circ}(2 \theta)$ and a count time of $5 \mathrm{~s}$ per step.

\section{Modeling the Kinetics of Lipolysis-Triggered Drug Precipitation}

The increased chemical potential of a supersaturated solution, in which the actual concentration of solubilized drug exceeds its solubility, is the driving force for drug precipitation. Precipitation can be described by two consecutive processes, i.e., nucleation and particle growth. The relationship between precipitation and supersaturation is reflected in the expressions describing the kinetics of these processes. According to the classical nucleation theory, the nucleation rate, $J\left(\mathrm{~m}^{-3} \mathrm{~s}^{-1}\right)$, is given by (43):

$J=\frac{d C_{p r}^{\prime}}{d t}=A^{\prime} S e^{-\frac{B}{l^{2} S}}$

where $C_{p r}^{\prime}$ is the number of nuclei formed per unit volume and $S$ is the degree of supersaturation given by $S=C / C^{*}$, with $C$ being the actual drug concentration and $C^{*}$ its solubility in the medium. Parameter $A^{\prime}\left(\mathrm{m}^{-3} \mathrm{~s}^{-1}\right)$ holds for the kinetic component of nucleation and is proportional to the number of nucleation-active centers. Its value differs between volume-diffusion-controlled and interface-controlled nucleation and is many orders of magnitude smaller for heterogeneous nucleation than for homogeneous nucleation (44). $B$ stands for the thermodynamic component of the process and is given by:

$B=16 \pi v_{0}^{2} \gamma^{3} / 3(k T)^{3}$

where $v_{0}$ is the molecular volume of the crystalline phase, $\gamma$ is the interfacial energy per unit area of the crystal, $k$ is the Boltzmann constant, and $T$ is the temperature. In the case of heterogeneous nucleation, the work of nucleation is reduced because of the lower interfacial energy, leading to smaller values of $B$.
The particle growth rate $G\left(\mathrm{~m} \cdot \mathrm{s}^{-1}\right)$ is often expressed heuristically as a power-law (45):

$G=k_{g}^{\prime}\left(C-C^{*}\right)^{g}$

where $k_{g}^{\prime} i$ s the particle growth coefficient, and $g$ is the order of particle growth.

To apply Eqs. 2 and 4 for modeling the kinetics of lipolysis-triggered drug precipitation, the changes in $C^{*}$ and $S$ as a function of time had to be considered. Fenofibrate solubility depends on the concentration of lipids in the medium and is therefore affected by the lipolysis, which changes the lipid composition over time. Hence, solubility was expressed as a function of time by a heuristic equation, which considered the contribution of different lipid species to solubility.

Considering the lipolysis kinetics on the one hand, and the specificity/activity of digestive enzymes (i.e., pancreatic lipase, co-lipase, and carboxyl ester hydrolase $(7,34,35,46))$ on the other, we subdivided the lipolysis of the formulation (containing TG, DG, and $\mathrm{MG}$ ) into two kinetic stages. TG and DG were assumed to be digested rapidly by the pancreatic lipase and co-lipase (first stage), while MG were digested slowly by the carboxyl ester hydrolase (second stage). The time point of transition between the first and second stages, $t_{x}$, was obtained graphically from the experimental FA titration curve. Based on the titration data and the stoichiometry of glyceride lipolysis, we calculated the moles of $\mathrm{TG}$ and $\mathrm{DG}\left(M_{1}(t)\right)$ digested into MG during the first stage and the moles of MG $\left(M_{2}(t)\right)$ digested during the second stage. The course of drug solubility in both stages was modeled using Eqs. 5 and 6:

$C_{m, t}^{*}($ stage 1$)=C_{m, 0}^{*} \bullet\left(1-\frac{M_{1}(t)}{M_{0}}\right)+C_{m, t x}^{*} \cdot\left(\frac{M_{1}(t)}{M_{0}}\right)$

$C_{m, t}^{*}($ stage 2$)=\left(C_{m, t x}^{*}-C_{m, 30}^{*}\right) \cdot\left(1-\frac{M_{2}(t)}{M_{t x}}\right)+C_{m, 30}^{*}$

where $C_{m, 0}^{*}, C_{m, t x}^{*}$, and $C_{m, 30}^{*}$ are the experimental drug solubility values in the lipolysis medium at times $0, t_{x}$, and $30 \mathrm{~min}$, respectively. $M_{0}$ is the molar quantity of TG and DG at $0 \mathrm{~min}$, and $M_{t x}$ is the molar quantity of MG in the medium at $t_{x}$. $M_{1}(t)$ and $M_{2}(t)$ were proportional to the logarithm of time. 
The kinetics of lipolysis-triggered drug precipitation was modeled by adapting Eqs. 2 and 4, and combining them with Eqs. 5 and 6. Three time intervals were defined that were delimited by $t_{0}$ (beginning of the experiment), $t_{n}$ (beginning of nucleation), $t_{g}$ (beginning of particle growth), and $t_{30}$ (end of the experiment). Thus, the model comprised the following system of ordinary differential equations:

If $t_{0}<t<t_{n}: \frac{d C_{p r}}{d t}=0$

if $t_{n}<t<t_{g}$ and $t<t_{x}: \quad \frac{d C_{p r}}{d t}=A S e^{-\frac{B}{l^{2} S}} \quad$ with $S=\frac{C}{C_{m, t}^{*}(\text { stage } 1)}$

if $t_{n}<t<t_{g}$ and $t>t_{x}: \quad \frac{d C_{p r}}{d t}=A S e^{-\frac{B}{h^{2} S}} \quad$ with $S=\frac{C}{C_{m, t}^{*}(\text { stage 2) }}$

if $t_{g}<t<t_{30}$ and $t<t_{x}: \frac{d C_{p r}}{d t}=k_{g}\left(C-C^{*}\right)^{g}$ with $C^{*}=C_{m, t}^{*}($ stage 1$)$

if $t_{g}<t<t_{30}$ and $t>t_{x}: \frac{d C_{p r}}{d t}=k_{g}\left(C-C^{*}\right)^{g}$ with $C^{*}=C_{m, t}^{*}($ stage 2)

$C_{p r}$ refers to the mass concentration of precipitated drug. During the induction time (between $t_{0}$ and $t_{n}$ ), no precipitation takes place (Eq. 7). During nucleation, the degree of supersaturation $S$ may be given by Eqs. 8 and 9 depending on the position of $t_{x}$ relative to $t_{n}$ and $t_{g}$. Analogously, during particle growth, the expression of solubility $C^{*}$ may depend on the position of $t_{x}$ with respect to $t_{n}$ and $t_{g}$ (Eqs. 10 and 11 ).

The values of parameters $A, B, k_{g}, g, t_{n}$, and $t_{g}$, were estimated by regressing this system of differential equations to the concentration data obtained by Raman spectroscopy. Mean $C_{p r}$ values of each drug load between 40 and $80 \mathrm{mg} / \mathrm{g}$ $(n=3)$ were used. Calculations were carried out using MATLAB. For parameter estimation we used the MATLAB optimization algorithm fminsearch, which is based on the simplex procedure. The ordinary differential equations were numerically solved using the MATLAB ode45 solver and the model quality was assessed based on the $R^{2}$ value and the root-mean-square error (RMSE).

\section{RESULTS}

\section{Formulation Characteristics}

The lipid-based system emulsified spontaneously within $10 \mathrm{~s}$, resulting in a fine emulsion with a droplet diameter of $39.5 \pm$ $0.3 \mathrm{~nm}$ and a PDI of $0.047 \pm 0.018$ (mean \pm standard deviation, $n=3$ ). No drug precipitation was measured $30 \mathrm{~min}$ after dispersion under non-digesting conditions. Fenofibrate solubility in the undigested lipolysis medium was $2.60 \pm$ $0.44 \mathrm{mg} / \mathrm{ml}$. However, upon addition of pancreatin extract, the solubility dropped rapidly to a value of $0.403 \pm$ $0.006 \mathrm{mg} / \mathrm{ml}$ within the first minute of digestion. By the end of the lipolysis experiment $(t=30 \mathrm{~min})$, solubility further decreased to $0.129 \pm 0.005 \mathrm{mg} / \mathrm{ml}$. On the other hand, fenofibrate solubility in the undispersed formulation, $C_{\text {form }}^{*}$, was $139.6 \pm 0.7 \mathrm{mg} / \mathrm{g}$.

Before lipolysis initiation, fenofibrate was below saturation, even at the highest drug load. As a consequence of the rapid decrease in drug solubility with formulation lipolysis, fenofibrate became supersaturated within the first minute of digestion. At the highest drug load $(80 \mathrm{mg} / \mathrm{g})$ the supersaturation ratio reached a value of $4.19 \pm 0.03$ ( $1 \mathrm{~min}$ of digestion), whereas at a drug load of $20 \mathrm{mg} / \mathrm{g}$, the corresponding supersaturation ratio was $1.04 \pm 0.02$.

Figure 2 shows the $\mathrm{NaOH}$ titration profile obtained from formulation lipolysis. Immediately after initiation of digestion, the hydrolysis was fast, while after approximately $1 \mathrm{~min}$ the rate had clearly decreased. No clear differences were observed between the lipolysis profiles of formulations with different drug loads.

Because not all FA were expected to be ionized at the $\mathrm{pH}$ of the assay, we employed the "back-titration" procedure. We obtained a back-titration factor of 1.09 for the formulation lipolysis, implying that $9 \%$ of the free FA were protonated at pH7.5 and, thus, not determined by direct titration. The lipolysis and back-titration of pure digestion medium (without SMEDDS) resulted in a correction factor of 1.77 .

\section{Detection of Drug Precipitation During Lipolysis}

\section{Ultracentrifugation Method}

To determine the amount of precipitated drug, we first applied ultracentrifugation for sample preparation, which appeared to be the most widespread method in this field of in vitro testing. Following ultracentrifugation, only two phases were obtained, i.e., an aqueous and a pellet phase. No oil phase was visually detectable with the given samples. This was, as expected, different from the samples that were not digested $(t=0)$, in which an oil phase was additionally observed. 


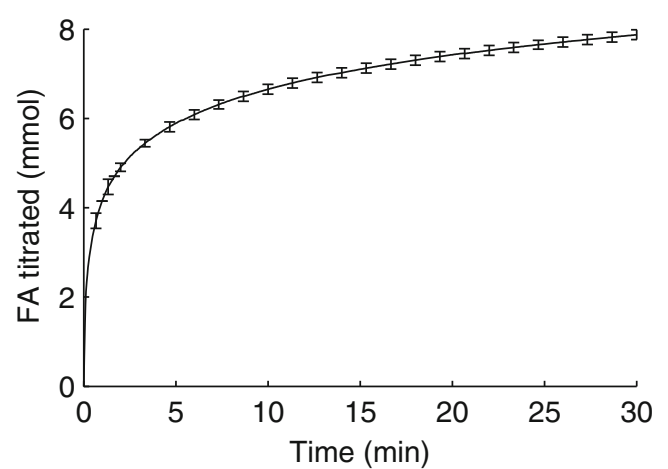

Fig. 2 Free FA originating from formulation digestion as a function of time. Values (means \pm standard deviation, $n=3$ ) were corrected for background lipolysis (pure lipolysis medium) and include additional FA detected by back-titration.

Figure 3a displays the fenofibrate precipitation profiles of formulations with varying drug loads, as obtained using ultracentrifugation. Drug precipitation started rapidly upon initiation of digestion and leveled off after approximately $2.5 \mathrm{~min}$ for formulations with drug loads of 40 to $80 \mathrm{mg} / \mathrm{g}$. Formulations with a lower drug load displayed some drug precipitation as well but the rates and extents were moderate.

Drug concentration in the aqueous phases decreased over time, in parallel to the increase in precipitation. After a 2.5 min digestion period, the concentrations of solubilized drug approached the equilibrium values, $C_{m, t}^{*}$, for all formulations with a drug load of $40-80 \mathrm{mg} / \mathrm{g}$.

We recorded the XRPD pattern of pure fenofibrate, of the pellet containing precipitated fenofibrate, and of a reference pellet. This reference was the pellet obtained after lipolysis of drug-free formulation, which was spiked with crystalline fenofibrate. As seen in Fig. 4, the angular range of peaks of the pellet containing precipitated drug corresponded to that of the reference pellet. Therefore, apparently, fenofibrate precipitated in the crystalline form during lipolysis.

\section{Nanofiltration Method}

The nanofiltration method allowed the determination of solubilized fenofibrate in the filtrate. Therefore, the concentration of precipitate was calculated as the difference between the total drug concentration and the amount of solubilized drug (Fig. 3b).

Similar to the ultracentrifugation method, we observed significant drug precipitation within $30 \mathrm{~min}$ of lipolysis with all formulations having a drug load of $40 \mathrm{mg} / \mathrm{g}$ and more. However, the time course was different, as there was an initial lag phase without precipitation before the drug finally started to precipitate out. Thus, for the SMEDDS with the
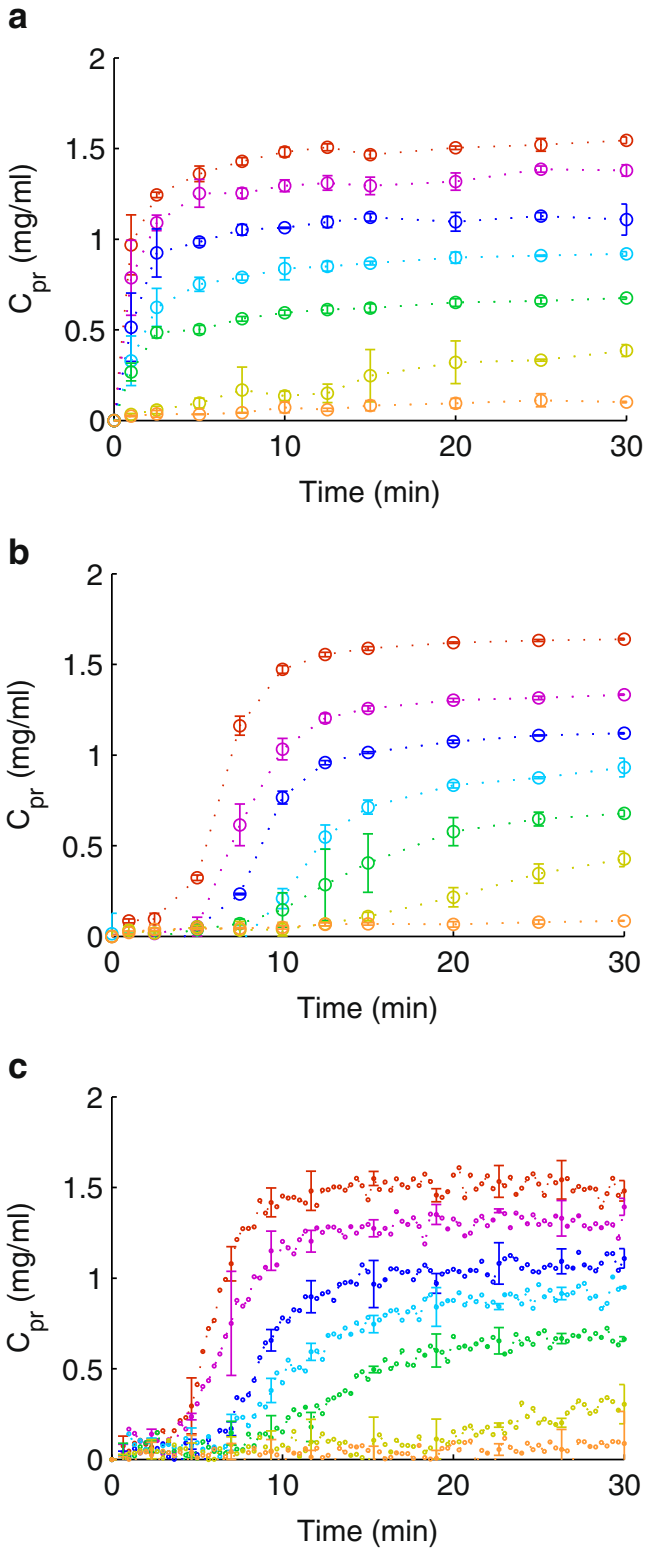

$\begin{array}{ll}\text { O } 80 \mathrm{mg} / \mathrm{g} & \text { 0 } 40 \mathrm{mg} / \mathrm{g} \\ \text { O } 70 \mathrm{mg} / \mathrm{g} & \text { ० } 30 \mathrm{mg} / \mathrm{g} \\ \text { O } 60 \mathrm{mg} / \mathrm{g} & \text { ○ } 20 \mathrm{mg} / \mathrm{g} \\ \text { O } 50 \mathrm{mg} / \mathrm{g} & \end{array}$

Fig. 3 Time course of fenofibrate precipitation during formulation lipolysis as detected (a) upon ultracentrifugation, (b) upon nanofiltration, and (c) with Raman spectroscopy. Each color represents a single drug loading, $C_{\text {form }}$. Values are expressed as means \pm standard deviation $(n=3)$.

highest drug load, precipitation started after approximately $2.5 \mathrm{~min}$, while for the formulation with a drug load of $30 \mathrm{mg} / \mathrm{g}$, drug precipitated after about $15 \mathrm{~min}$. The concentration of solubilized drug did not significantly change within the first minute of digestion for each drug load $(p>0.05)$. Finally, only minimal precipitation was observed in the formulation with a $20 \mathrm{mg} / \mathrm{g}$ drug load. 


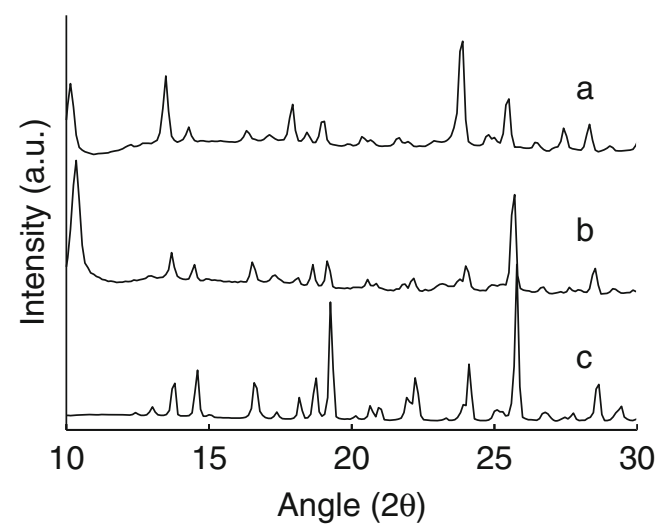

Fig. 4 XRPD pattern of the pellet phase obtained upon ultracentrifugation and resulting from (a) the formulation with $80 \mathrm{mg} / \mathrm{g}$ fenofibrate, (b) the pellet phase of drug-free formulation spiked with crystalline fenofibrate, and (c) pure crystalline fenofibrate.

\section{Raman Spectroscopy}

The Raman spectra of fenofibrate in the crystalline and solubilized forms displayed a characteristic pattern, as seen in Fig. 5a. Unprocessed spectra are depicted, which were recorded during the lipolysis experiment at different time points (drug load: $70 \mathrm{mg} / \mathrm{g}$ ). Moreover, the Raman spectra of crystalline fenofibrate and of the drug-free lipolysis medium are shown. The spectra recorded after $40 \mathrm{~s}, 5 \mathrm{~min}$, and $30 \mathrm{~min}$ of lipolysis displayed noticeable variations in the range of $1132-1164 \mathrm{~cm}^{-1}$ and $1550-1663 \mathrm{~cm}^{-1}$.

A PLS model was built for the concentration of crystallized fenofibrate based on a set of 42 spectra, as detailed in Table I. The reference values were mostly selected from concentrations and time points that demonstrated consistent results in both off-line methods (i.e., ultracentrifugation and nanofiltration). The concentrations in samples with a drug load of $50-$ $80 \mathrm{mg} / \mathrm{g}$, assayed at 25 and $30 \mathrm{~min}$ of digestion, were consistent between the two methods and were therefore used for model calibration of the corresponding spectra. To further include spectra of digests without precipitate, we relied on the data from the nanofiltration method. According to these results, the concentration of solubilized drug after $1 \mathrm{~min}$ of lipolysis was not significantly different from the concentration before initiation of lipolysis ( $p>0.05$ in all cases). This observation indicates that no precipitation occurred during the first minute of digestion. Therefore, for model calibration we selected the earliest spectrum (i.e., the spectrum that was acquired $40 \mathrm{~s}$ after lipolysis initiation) of each formulation with a drug load of $20-70 \mathrm{mg} / \mathrm{g}$ and set $C_{p r}$ equal to zero. The $80 \mathrm{mg} / \mathrm{g}$ drug load was excluded from this part of the calibration.

We evaluated different methods for data preprocessing. Two scatter-correction methods (MSC, SNV) and a derivative method (SG) were compared (38-40). Moreover, three different spectral ranges were considered for model a

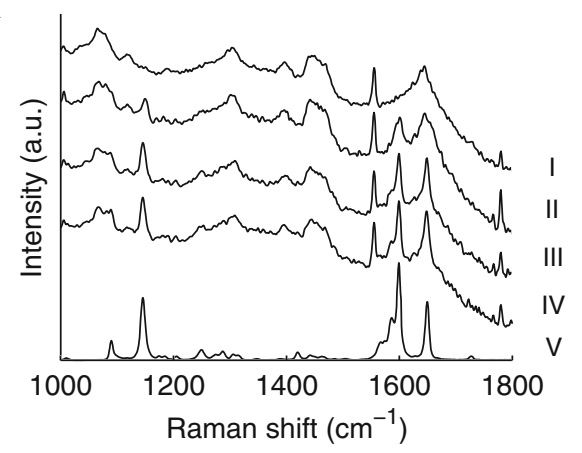

b

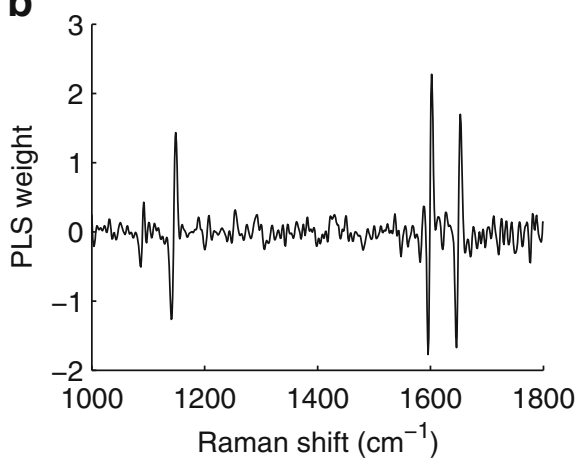

C

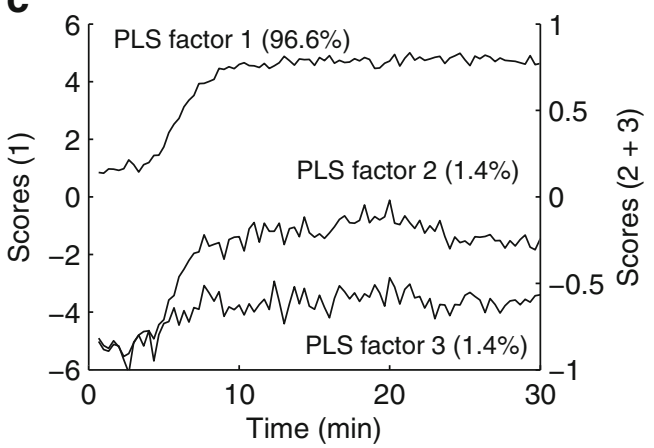

Fig. 5 Part (a) depicts the unprocessed Raman spectra of drug-free lipolysis medium (I), lipolysis medium with fenofibrate after 40s (II), 5 min (III), and $30 \mathrm{~min}$ (IV) digestion periods, and pure fenofibrate ( $\mathrm{V}$ ). Plots of the weights of the first PLS factor (b) and scores over time of all three PLS factors used in the PLS model (c). The numbers in (c) represent the variance captured in the corresponding PLS factor. All plots are offset for clarity.

calibration. Table II compares the performance of calibration models that were obtained using the different preprocessing methods and spectral ranges. The best

Table I Calibration Set as Used for Quantitative Application of Raman Spectroscopy

\begin{tabular}{llll}
\hline$C_{\text {form }}(\mathrm{mg} / \mathrm{g})$ & $\begin{array}{l}\text { Time span of } \\
\text { acquisition (s) }\end{array}$ & $C_{p r}(\mathrm{mg} / \mathrm{ml})$ & $\begin{array}{l}\text { Total number of } \\
\text { calibration spectra }\end{array}$ \\
\hline $20,30,40,50,60,70$ & $20-40$ & 0 & 18 \\
$50,60,70,80$ & $1480-1500$ & $C_{p r}{ }^{a}$ & 12 \\
$50,60,70,80$ & $1780-1800$ & $C_{p r}{ }^{a}$ & 12 \\
\hline
\end{tabular}

${ }^{a}$ Results obtained using the ultracentrifugation method 
calibration model was attained with the SG algorithm $\left(1^{\text {st }}\right.$ derivative) and a spectral range between 1000 and $1800 \mathrm{~cm}^{-1}$. Three PLS factors described $99.4 \%$ of the spectral variation, with an $R^{2}$ value of 0.994 , while the first, second, and third factors accounted for $96.6 \%, 1.4 \%$, and $1.3 \%$ of the variation, respectively. Only the first PLS factor is shown in Fig. 5b, as it accounted for most of the variation. Herein, three ranges with a high PLS weight were identified, which corresponded to the peaks of the crystalline fenofibrate. The second and the third factors contributed to the model variation to a lower extent. However, they may account for residual non-linearity in the data set as seen in Fig. 5c. The cross-validation procedure yielded a $Q^{2}$ value of 0.972 and an RMSECV of $0.111 \mathrm{mg} / \mathrm{ml}$, indicating a robust calibration model.

Finally, we applied this calibration model to all other Raman spectra to determine the precipitation profiles over a 30 min digestion period. Figure $3 \mathrm{c}$ shows that fenofibrate precipitated after an initial lag time in all formulations with drug loads of more than $30 \mathrm{mg} / \mathrm{g}$. Minimal drug precipitation was observed for the formulation with a drug load of $20 \mathrm{mg} / \mathrm{g}$.

In summary, the comparison of fenofibrate precipitation profiles (Fig. 3a, b, and c) demonstrated an excellent agreement between the nanofiltration and the spectroscopic methods. Both methods suggested that drug precipitated only after an initial lag phase. This was in contrast to the ultracentrifugation method, which indicated precipitation almost immediately after digestion started.

\section{Modeling the Kinetics of Lipolysis-Triggered Drug Precipitation}

The high temporal resolution of in-line Raman spectroscopy encouraged a more detailed analysis of drug precipitation kinetics. The theories of nucleation and growth (Eqs. 2 and 4) were applied to model the course of lipolysis-triggered drug precipitation. Before lipolysis initiation, drug concentration in the lipolysis medium was below saturation. Enzymatic digestion of lipids resulted in a diminished drug solubilization, which in turn induced supersaturation and led to drug precipitation. Because the drug solubility changed continuously through ongoing lipolysis, an expression for time-dependent solubility was introduced.

The progress of lipolysis is reflected by the amount of FA titrated as a function of time (Fig. 2). These data show that lipolysis occurred rapidly at first and then slowed down considerably. By drawing two straight lines through the data points between 0 and $40 \mathrm{~s}$, and between 16.6 and $30 \mathrm{~min}$, we found the intersection point, $t_{x}$, at $82.1 \mathrm{~s}$. This time point was used to define the transition of the initial rapid lipolysis stage to the second, slow, lipolysis stage. Based on the titration data and the stoichiometry of hydrolysis, we calculated the amount of glycerides that were apparently hydrolyzed in these two stages (Fig. 6a), and compared it with the amount of formulation lipids. The good agreement between experimental data (Fig. 6a), and the formulation composition (Table III) supports the view that the first stage corresponded primarily to the hydrolysis of TG and DG, whereas the second stage was attributable to the hydrolysis of MG. The latter value included MG, those present in the formulation (Imwitor ${ }^{\circledR} 988$ ) and those generated from the lipolysis of sn-1,3 DG. In contrast, sn-2 MG resulting from the digestion of TG were assumed not to be further hydrolyzed (46). With respect to the different enzyme activities, we expected rapid digestion of DG and TG by the pancreatic lipase and co-lipase, whereas the activity of the carboxyl ester hydrolase is known to be comparatively low $(34,35)$. This difference in lipolysis rates was in good agreement with our observations, as DG and TG were digested considerably faster than MG. The amount of digested
Table II PLS Analysis Results of Raman Spectroscopy
aParameters used in the SavitzkyGolay algorithm: window size 10 points, $1^{\text {st }}$ derivative, $2^{\text {nd }}$

polynomial.

'Spectral ranges:

$A=500-1800 \mathrm{~cm}^{-1}$

$\mathrm{B}=1000-1800 \mathrm{~cm}^{-1}$;

$C=|132-1| 64, \mid 550-1615$,

$1635-1663 \mathrm{~cm}^{-1}$

\begin{tabular}{lllllll}
\hline Pre-processing method & $\begin{array}{l}\text { Spectral } \\
\text { range }^{b}\end{array}$ & PLS factors & $R^{2}$ & $Q^{2}$ & $\begin{array}{l}\text { RMSEC } \\
(\mathrm{mg} / \mathrm{ml})\end{array}$ & $\begin{array}{l}\text { RMSECV } \\
(\mathrm{mg} / \mathrm{ml})\end{array}$ \\
\hline None & A & 5 & 0.985 & 0.969 & 0.0142 & 0.113 \\
& B & 4 & 0.985 & 0.959 & 0.0104 & 0.129 \\
SNV & C & 3 & 0.979 & 0.960 & 0.0501 & 0.112 \\
& A & 3 & 0.984 & 0.907 & 0.0298 & 0.194 \\
MSC & B & 3 & 0.988 & 0.962 & 0.0199 & 0.152 \\
& C & 4 & 0.953 & 0.962 & 0.0108 & 0.124 \\
& A & 4 & 0.985 & 0.958 & 0.0129 & 0.132 \\
SG & B & 3 & 0.978 & 0.958 & 0.0133 & 0.132 \\
& C & 3 & 0.982 & 0.966 & 0.0158 & 0.117 \\
& A & 3 & 0.993 & 0.971 & 0.0083 & 0.117 \\
& B & 3 & 0.994 & 0.972 & 0.0079 & 0.111 \\
& C & 3 & 0.990 & 0.969 & 0.0099 & 0.111 \\
\hline
\end{tabular}



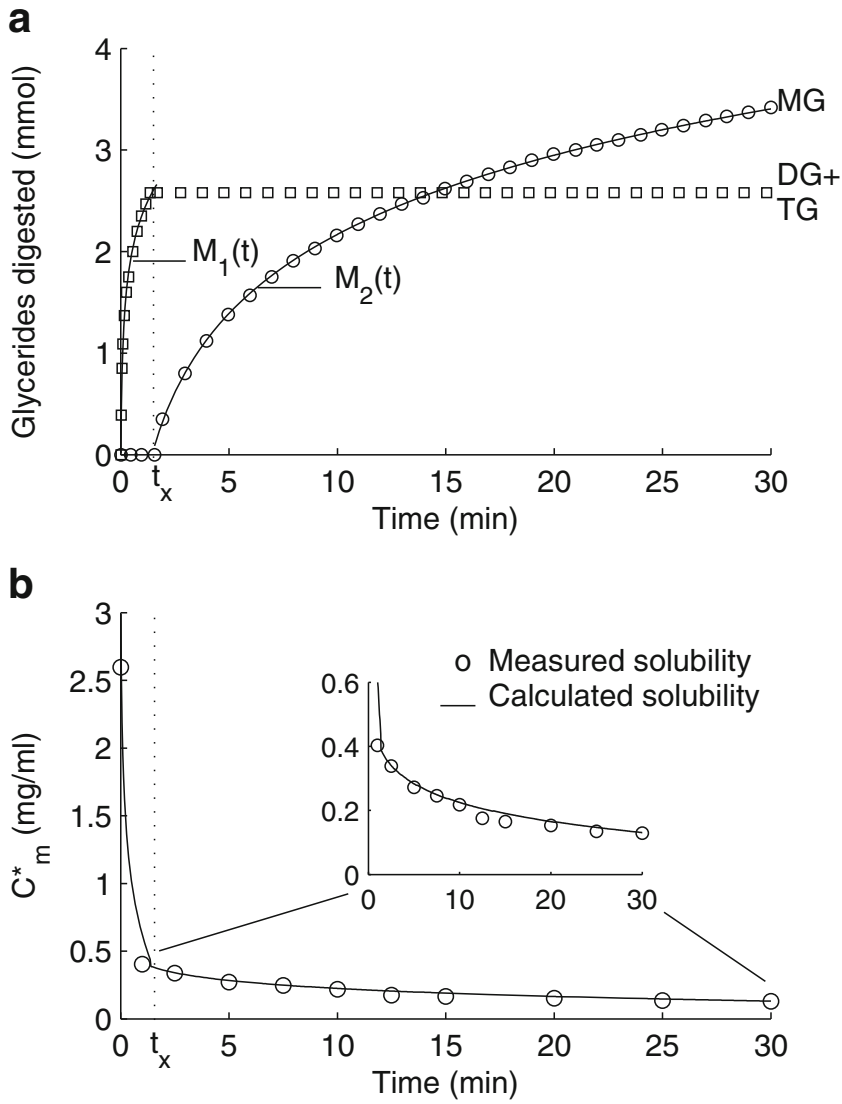

Fig. 6 TG and DG digestion (white square) and sn-I MG digestion (white circle) over time, as calculated based on titrated FA and the stoichiometry of glyceride hydrolysis according to the literature (a). The solid lines represent the logarithmic functions of time fitted to these time courses $\left(M_{1}(t)=\right.$ $0.57 \cdot \ln (t)+2.37$ and $M_{2}(t)=1.12 \cdot \ln (t)-0.419$, respectively). (b) Fenofibrate solubility in the lipolysis medium as determined experimentally at different lipolysis times (circles) and as calculated from Eqs. 5 and 6 (solid line).
TG and DG $\left(M_{1}(t)\right)$ and of digested MG $\left(M_{2}(t)\right)$ was found to be well described by a logarithmic function of time $\left(R^{2}>\right.$ 0.99), as shown by the fitted lines in Fig. 6 a.

These findings of lipolysis kinetics were used to propose a mathematical expression for solubility as a function of time. It was assumed that two phases contributed to overall solubility. An oil phase consisted of TG and DG, whereas a colloidal phase consisted primarily of MG. Both contributions to the total solubility were considered additively. Therefore, drug solubility in the first and second stages of lipolysis was given by Eqs. 5 and 6, respectively. Figure 6b demonstrates that the curve, which was calculated (not fitted) using these equations, accurately reflected the experimental solubility values. The drug solubility at $82.1 \mathrm{~s}$ was found by interpolation and corresponded to a value of $0.39 \mathrm{mg} / \mathrm{ml}$. It must be mentioned that no single mathematical expression was able to describe adequately the change in drug solubility over the entire time course of lipolysis.

Equations 2 and 4 were combined with Eqs. 5 and 6 to give the system of differential equations (Eqs. 7-11), which was fitted to the concentration data obtained by Raman spectroscopy. The model was found to excellently describe the experimental concentrations of precipitated drug $\left(R^{2}>0.975\right)$ at all drug loads (Fig. 7). Table IV details the values of $A, B, k_{g}, g, t_{n}$, and $t_{g}$ that were estimated from this optimization. The nucleation parameters $A$ and $B$ had comparable values for all studied drug loads, while the nucleation induction time, $t_{n}$, increased with decreasing drug load. The growth parameters exhibited values within a similar range for each drug load. No fitting was carried out for the smallest drug loads of 20 and $30 \mathrm{mg} / \mathrm{g}$ because of the large scatter in Raman data.

Table III Estimation of the Amount of FA Liberated from $2.6 \mathrm{~g}$ SMEDDS According to Assumptions Inferred from the Literature $(34,35,46)$ and Maximal Amount of Hydrolysable Ester Bonds in $2.6 \mathrm{~g}$ SMEDDS

\begin{tabular}{|c|c|c|c|c|c|}
\hline Excipient & $\begin{array}{l}\text { Composition in SMEDDS } \\
(\% \mathrm{w} / \mathrm{w})\end{array}$ & $\begin{array}{l}\text { Excipient composition } \\
(\% w / w)\end{array}$ & $\begin{array}{l}\text { Excipient in } 2.6 \mathrm{~g} \\
\text { formulation }(\mathrm{mmol}){ }^{b}\end{array}$ & $\begin{array}{l}\text { FA liberation according } \\
\text { to the literature }{ }^{a}(\mathrm{mmol})\end{array}$ & $\begin{array}{l}\text { Max. available FA } \\
(\mathrm{mmol})\end{array}$ \\
\hline Miglyol 812 & 40 & $100(\mathrm{TG})$ & 2.01 & $4.03^{\dagger}$ & 6.04 \\
\hline \multirow[t]{3}{*}{ Imwitor $® 988$} & \multirow[t]{3}{*}{20} & $54.6(M G)$ & 1.45 & $1.45^{\ddagger}$ & 1.45 \\
\hline & & 38.0 (DG) & 0.58 & $0.58^{\dagger}$ and $0.58^{\ddagger}$ & 1.16 \\
\hline & & 7.1 (TG) & 0.077 & $0.15^{+}$ & 0.23 \\
\hline Cremophor ${ }^{\circledR}$ RH 40 & 40 & $100(\mathrm{TG})$ & 0.39 & $0.09^{\ddagger}$ & 1.16 \\
\hline
\end{tabular}

${ }^{a}$ TG in Miglyol 812 and Imwitor ${ }^{\circledR} 988$ are digested to sn-2 MG and 2 FA; sn- I,3 DG in Imwitor ${ }^{\circledR} 988$ are digested to sn- I MG and I FA, and then to glycerol and I FA; sn- I MG in Imwitor ${ }^{\circledR} 988$ are digested to glycerol and FA; Cremophor ${ }^{\circledR}$ RH 40 was assumed to be hydrolyzed by $7.5 \%$ (w/W) according to Cuiné et al. (5). Digestion of TG to sn- I,2 DG and sn-2 MG and of sn- I,3 DG to sn- I MG was assumed to occur rapidly ( ${ }^{\dagger}$, first stage), while the digestion of Cremophor ${ }^{\circledR} \mathrm{RH} 40$ and of sn- I MG to glycerol and FA was supposed to occur slowly ${ }^{\ddagger}$, second stage)

${ }^{b}$ Average molecular weights $(\mathrm{g} / \mathrm{mol}$ ) used for calculation: 516.8 (Miglyol 8/2), 196.2 (MG in Imwitor 988), 339.4 (DG in Imwitor $\AA$ 988), 482.6 (TG in Imwitor ${ }^{\circledR}$ 988), 2699 (Cremophor ${ }^{\circledR}$ RH 40 (5)) 
Fig. 7 Concentration profile of precipitated fenofibrate as measured with Raman spectroscopy (circles) and as obtained from mathematical modeling (solid lines). Each plot represents the result of a single drug loading and the circles are mean values of three experiments.
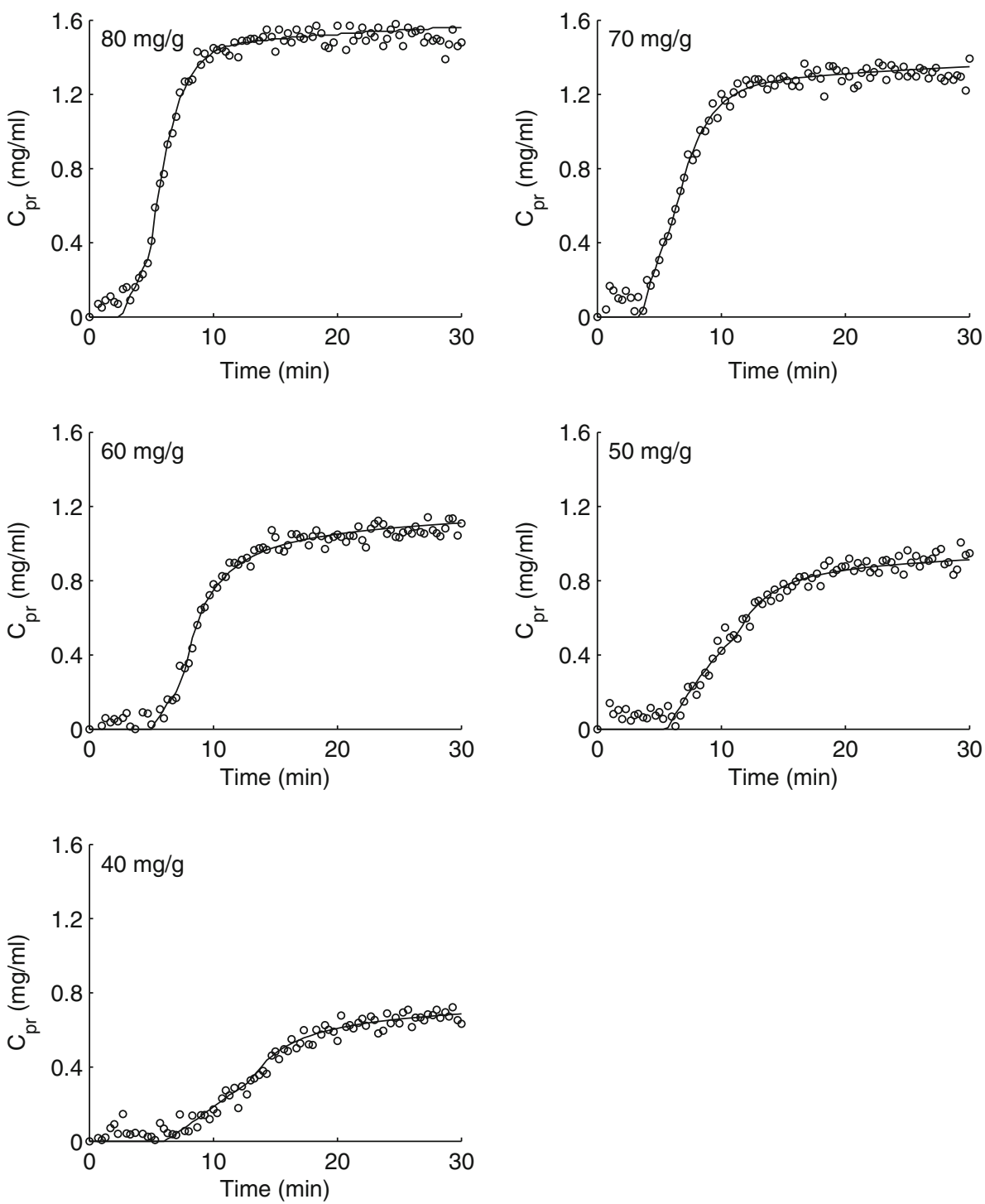

\section{DISCUSSION}

\section{Detection of Drug Precipitation During In Vitro Lipolysis}

The fate of a LBDDS in the gastrointestinal tract is decisive for drug absorption and, therefore, for the performance of the formulation. Poorly water-soluble drugs often precipitate from lipid-based systems already upon dispersion in aqueous fluids. Such potentially inferior systems can be identified early in formulation screening using simple in vitro dilution and dispersion tests (47). Other formulations exhibit drug precipitation in the course of formulation digestion. The analysis of such lipolysis-triggered drug precipitation was the main focus of the present work.

Traditionally, drug solubilization during in vitro lipolysis is analyzed using methods that rely on a sampling regime. Accordingly, aliquots of the digest are drawn
Table IV Results of Parameter Estimation of Drug Precipitation Kinetics

\begin{tabular}{lllllllll}
\hline$C_{\text {form }}(\mathrm{mg} / \mathrm{g})$ & $A\left(10^{-3} \mathrm{mg} \cdot \mathrm{ml}^{-3} \mathrm{~s}^{-1}\right)$ & $B$ & $t_{n}(\mathrm{~s})$ & $t_{\mathrm{g}}(\mathrm{s})$ & $k_{\mathrm{g}}\left(10^{-3} \mathrm{~s}^{-1}\right)$ & $g$ & $R^{2}$ & $\mathrm{RMSE}(\mathrm{mg} / \mathrm{ml})$ \\
\hline 80 & 2.0 & 6.1 & 149.0 & 291.0 & 9.0 & 0.8 & 0.990 & 0.052 \\
70 & 2.9 & 4.8 & 210.5 & 395.2 & 7.3 & 0.9 & 0.987 & 0.052 \\
60 & 2.0 & 6.6 & 300.2 & 478.7 & 9.0 & 1.4 & 0.992 & 0.041 \\
50 & 2.8 & 6.3 & 335.9 & 671.7 & 8.4 & 1.2 & 0.978 & 0.051 \\
40 & 1.6 & 6.2 & 362.0 & 824.8 & 8.2 & 1.3 & 0.976 & 0.040 \\
\hline
\end{tabular}


from the lipolysis medium at defined time points and the concentration of solubilized and/or precipitated drug is usually measured following ultracentrifugation. This approach has so far provided fundamental knowledge on the lipolysis of lipid-based systems as well as the in vitro fate of poorly water-soluble drugs. However, real-time monitoring seems to be essential to further analyze the dynamic changes of the formulation and to study effects of drug supersaturation.

The first aim of this study was to evaluate Raman spectroscopy for real-time monitoring of drug precipitation during in vitro lipolysis. A model SMEDDS containing fenofibrate was digested using a common lipolysis test (7). The application of Raman spectroscopy to in vitro lipolysis represented a particularly challenging case for monitoring of crystallization. While classical crystallization monitoring in chemical processes usually involves high drug concentrations and a constant medium, the present application of Raman spectroscopy had to cope with low analyte concentrations in a changing digestion medium. The hydrolysis products interfered with the drug-related Raman signal and had to be considered in the interpretation of spectra. Moreover, the two off-line methods (ultracentrifugation and nanofiltration) revealed some conflicting results, which led to an even more challenging development of a robust calibration model. Hence, an accurate selection of instrumental parameters, chemometric procedures, and reference measurements was required.

The time courses of drug precipitation obtained from the two off-line sample preparation methods were dissimilar, particularly in the initial stage of precipitation. This difference was likely due to additional drug precipitation occurring during sample ultracentrifugation. Even though ultracentrifugation was proven to separate the solid, aqueous, and oil phases efficiently (11), the influence of sample preparation on drug precipitation can barely be avoided. First, there was an unavoidable time delay of up to $30 \mathrm{~min}$ between sample removal and ultracentrifugation. Second, we allowed ultracentrifugation to proceed for $90 \mathrm{~min}$. This step usually takes a shorter time (30 min (11)), but we deliberately selected an extreme condition (90 min (48)) to simulate a worst-case sample preparation. These steps introduced time delays during which the drug could further precipitate. Therefore, the results of the ultracentrifugation method might not provide the actual kinetic concentration but rather an advanced stage of drug precipitation. This assumption was supported by the observation that drug concentrations in the aqueous phase, as measured upon ultracentrifugation, were almost equal to the corresponding solubilities. In contrast, the nanofiltration method allowed a more rapid separation of the liquid phase. This method has proven to effectively separate undissolved drug from colloidal drug solutions in biorelevant release tests (37).
The selection of calibration spectra and reference measurements relied on these previous findings. According to the nanofiltration results, the concentration of solubilized drug did not significantly change within the first minute of lipolysis. This observation proved that fenofibrate did not precipitate instantaneously when lipolysis was started, but only after an initial lag phase. It was therefore justified to include the earliest spectra and the corresponding nanofiltration result within the calibration set. Between 25 and $30 \mathrm{~min}$ of digestion, the nanofiltration and ultracentrifugation methods yielded consistent results and thus the corresponding spectra and reference values were included in the calibration set. Finally, between $40 \mathrm{~s}$ and 25 min there was a lack of reliable reference measurements, hence those spectra were excluded from the set of calibration spectra.

The generation of lipolysis products led to a notable increase in medium turbidity. This light scattering originated mostly from FA precipitation in the presence of calcium and from precipitated drug. To reduce particlesize effects, we selected adequate sampling optics consisting of a large spot-size Raman probe (49). Furthermore, we evaluated different pre-processing algorithms for the entire set of Raman spectra prior to PLS regression analysis. This was necessary to correct for light scattering and residual particle-size effects (40). We found that an SG smoothing and differentiation filter ( $1^{\text {st }}$ derivative) was best for reducing this systematic variation. Residual sources of nonlinearity were finally corrected by a three-component PLS regression model, while cross-validation demonstrated good robustness.

After spectra pre-processing and calibration, we applied the resultant quantification model to the entire data set. The real-time data demonstrated that drug started to precipitate after a lag phase of 3 to $6 \mathrm{~min}$, while this period decreased with increasing drug load. Interestingly, there was excellent agreement between the course of drug precipitation obtained from Raman spectroscopy and from the nanofiltration method, even though only spectra from the initial and final time points were included in the calibration set.

Raman spectroscopy demonstrated clear advantages compared with the traditional ultracentrifugation method. The main issue in the latter method was the time delay caused by sample preparation. The application of benchtop centrifugation instead of ultracentrifugation, as proposed in a recent publication (11), could reduce this time delay. However, temporal resolution is still better using an in situ method such as Raman spectroscopy.

In contrast to ultracentrifugation, conventional Raman spectroscopy may be barely able to determine drug solubilization in the aqueous and the oil phases separately. However, the authors of a recent study suggested that the concentration of solubilized drug in the entire medium (and not only in the aqueous phase) may be the relevant measure for predicting 
intestinal permeation of a drug (14). In this respect Raman spectroscopy can provide the relevant data for estimating the absorbable dose fraction. Even though Raman spectroscopy was applied to the quantification of crystalline drug in the present case, the technique would also be able to quantify amorphous drug precipitation. This is certainly an advantage over, for example, in situ XRPD. However, the combination of XRPD and Raman spectroscopy is certainly of interest for studying transient polymorphic changes (50).

\section{Kinetics of Lipolysis-Triggered Drug Precipitation}

The second aim of the study was to determine the kinetics of drug precipitation during formulation lipolysis. Modeling precipitation kinetics may offer advantages in the biopharmaceutical characterization of lipid-based systems. Indeed, the amount of drug available for absorption does not only depend on whether or not the drug precipitates in the gastrointestinal lumen. Besides improving drug solubility, lipid-based formulations can increase bioavailability by the temporary stabilization of a drug in the supersaturated state. Although it is in a metastable condition, this induction time is particularly relevant for compounds with good permeability. Indeed, supersaturation generates an increased flux across the intestinal membrane and precipitation in vivo may be reduced or even be absent. This awareness is crucial for formulation development. Drug supersaturation and the solid-state properties of a precipitate are often neglected during industrial screening tests of lipid-based formulations. Such candidate systems generally drop out of screening programs and, consequently, there is a risk of excluding candidate formulations that may still exhibit sufficient oral availability in vivo. Physiologically based pharmacokinetic (PBPK) models are valuable tools for predicting the formulation behavior in vivo on the basis of characteristics that are measured in vitro (47). These models consider drug release and absorption as a dynamic interplay to predict the bioavailability of a drug. However, to obtain a precise estimation, PBPK modeling requires accurate prediction of supersaturation and potential drug precipitation during formulation dispersion and/or lipolysis.

\section{Nucleation and Growth Model}

We presented a nucleation and growth model that considered the dynamic changes in drug solubility during formulation digestion. The new model successfully described the data of lipolysis-triggered fenofibrate precipitation and yielded reasonable parameter values. The kinetic and thermodynamic pre-factors of the nucleation rate, $A$ and $B$, respectively, were consistent for different drug loads and are in good agreement with the literature (44). For such comparison, it has to be noted that the pre-factor $A$ is here expressed as a mass concentration per unit time. The growth constant $k_{g}$ and the exponent $g$ were also in accordance with previously reported values (45).

When comparing the kinetics of drug precipitation with the kinetics of formulation lipolysis, we observed a non-linear correlation between the two variables (Fig. 8). This was interesting in relation to the findings of Sassene et al., who observed linearity between precipitated cinnarizine and titrated FA using a self-microemulsifying formulation (24). In this previous study, continuous calcium addition and the different sample preparation method (ultracentrifugation) may have influenced the observed kinetics. To clarify such effects, more studies that compare the different lipolysis tests using the same drug formulation are certainly needed.

Our study indicates that the nucleation time $t_{n}$ decreased with increasing drug load, i.e., with higher levels of supersaturation, in good agreement with expectation, as the degree of supersaturation generally decreases the induction period (45). This dependence is highly relevant for selecting the adequate dose strength. Accordingly, the administration of a single lipid-based capsule with high dose strength might result in a different pharmacokinetic profile compared with the administration of multiple units at lower dose strength. These considerations are in good agreement with a recent attempt to study the effects of drug loading on the lipolysis of lipid-based formulations (51).

Several studies used the in vitro digestion model to predict formulation performance in viwo. However, limited correlation between in vitro solubilization and in vivo exposure was often apparent. For instance, in an attempt to prove supersaturation stabilization in SEDDS with polymers, Anby et al. showed a clearly beneficial effect of polymer addition upon in vitro digestion, but no correlation was found with in vivo data (4). Other studies showed an agreement between in vitro and in viwo data, but primarily as a rank-order correlation (48,52-55). An improved characterization of drug supersaturation, precipitation, and re-dissolution might be the key to better understand the pharmacokinetic processes in vivo. To this end, real-time

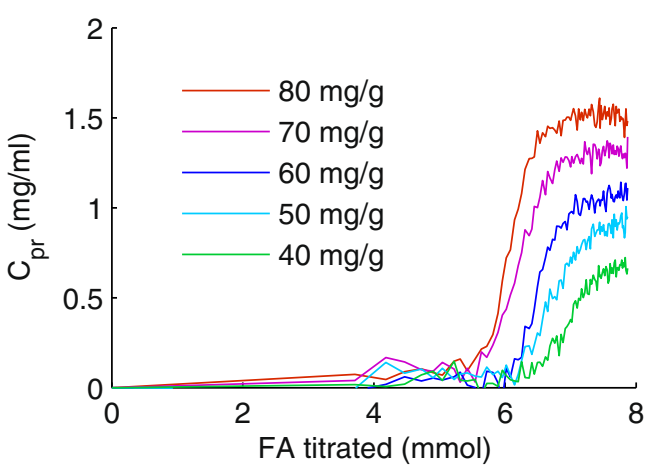

Fig. 8 Fenofibrate precipitation as a function of titrated FA. Each color represents a single drug loading and is the mean value of three experiments. 
Raman spectroscopy, together with mathematical modeling of lipolysis-triggered drug precipitation, could play an important role. These techniques may become part of future drug absorption modeling and improve the predictivity of in vitro-in vivo correlations.

\section{Drug Solubility as a Function of Time}

An important part of the presented nucleation and growth model was to consider the change in drug solubility as a function of time. The proposed heuristic function was in line with previous studies that proved a direct correlation between drug solubility and the concentration of formulation components and digestion products $(18,56,57)$. However, some discussion is needed to explain why the present heuristic approach was proposed.

The pancreatin extract consisted of a mixture of digestive enzymes and co-enzymes, most importantly pancreatic lipase and co-lipase, as well as carboxyl ester hydrolase $(58,59)$. These enzymes exhibit different lipolytic activities and substrate specificities, as described previously $(34,35,46,60)$. These properties of lipolytic enzymes and the composition of the model formulation suggested that lipolysis occurred in two stages. Pancreatic lipase and colipase are known to rapidly hydrolyze TG and DG at the sn1,3 position, yielding sn-2 $\mathrm{MG}$ and FA as digestion products. It is assumed that this substrate specificity is due to the high affinity of the pancreatic lipase and co-lipase to insoluble lipids $(34,35)$. In contrast, the second stage was likely characterized by the digestion of MG, which is catalyzed mostly by carboxyl ester hydrolase. This enzyme has high affinity to lamellar structures and micelles containing MG and its lipolytic activity is lower than that of pancreatic lipase (34,35). Although the regioselectivity of carboxyl ester hydrolase is still a debated topic, it is assumed to cleave MG preferentially at the sn-1 position (60,61). Sek et al. quantified the digestion products of medium-chain and long-chain glycerides upon in vitro digestion, using the same experimental conditions as in the present study (46). Interestingly, sn-2 MG were weakly digested by porcine digestive enzymes, whereas sn-1 MG were hydrolyzed to a large extent. For this reason, we assumed that primarily sn-1 MG were digested in the second stage of lipolysis.

The amounts of DG and TG hydrolyzed during the first stage of lipolysis (as obtained from the titration data) were in good agreement with the nominal amount of lipids that was present in the formulation. For the second stage of lipolysis, we expected sn-1 MG and a small fraction of Cremophor ${ }^{\circledR} \mathrm{RH}$ 40 to be hydrolyzed (Table III, FA liberation according to the literature). Interestingly, considering these assumptions, the amount of titrated FA exceeded the expected FA liberation. This observation could originate from higher Cremophor ${ }^{\circledR}$ RH 40 hydrolysis than the reported fraction of $7.5 \%$ (5). This value was obtained from the in vitro lipolysis of pure excipient and it might not be the same for Cremophor ${ }^{\circledR}$ RH 40 digestion in a lipid mixture. Care is needed when assuming that a single excipient is digested equally to when it is present in combination with an oil phase in a formulation. The situation is particularly complex for surfactants, as they are known to act as substrates and as inhibitors of lipolytic enzymes (5). A further explanation for the observed lipolysis degree is that some sn-2 MG were further digested. Mattson and Volpenhein observed that a small fraction of sn-2 MG exhibited a non-enzymatic isomerization to sn-1 MG (62). In line with their observation, it seems possible that a small amount of sn-2 MG exhibited isomerization and was further digested.

As a result, the proposed equation for fenofibrate solubility as a function of time was in very good agreement with the experimental solubility values. DG and TG were the most lipophilic glycerides present in the formulation. Therefore, the removal of these compounds was correlated with a pronounced decrease in fenofibrate solubilization. In contrast, we measured a less pronounced decrease during the second stage of lipolysis because of the smaller contribution of MG to solubilization.

\section{CONCLUSIONS}

Real-time analytics is fundamental to gain an improved understanding of the highly dynamic processes of formulation digestion. In this study we evaluated the quantitative application of Raman spectroscopy for real-time monitoring of drug precipitation during in vitro lipolysis. The method was compared with two off-line procedures, which were based on a sampling regime. Raman spectroscopy measured fenofibrate precipitation with a high temporal resolution and minimal experimental bias. In contrast, care is needed when determining the kinetics of drug precipitation by methods that require sample preparation. Indeed, the lag time between sample removal and analysis could lead to further drug precipitation.

The precipitation profiles obtained by Raman spectroscopy were used to model the kinetics of lipolysis-triggered drug precipitation. We introduced a mathematical model, which was based on theoretical equations of nucleation and growth by considering solubility changes over time. This model provided an excellent prediction of fenofibrate crystallization during lipolysis. The drug began to precipitate after an initial lag phase of supersaturation. The temporary supersaturation is not only of theoretical interest. This metastable state is particularly important for poorly water-soluble compounds with high permeability, i.e., when the process of drug precipitation occurs in a similar time span as drug absorption.

The proposed model for lipolysis-triggered drug precipitation may be part of more complex pharmacokinetic models. Physiologically based drug absorption models are particularly 
interesting in this aspect. More refined mechanistic modeling of this type will ultimately help to better predict the fate of a LBDDS in vivo.

\section{REFERENCES}

1. O’Driscoll CM, Griffin BT. Biopharmaceutical challenges associated with drugs with low aqueous solubility - the potential impact of lipidbased formulations. Adv Drug Del Rev. 2008;60(6):617-24.

2. Porter CJH, Trevaskis NL, Charman WN. Lipids and lipid-based formulations: optimizing the oral delivery of lipophilic drugs. Nat Rev Drug Discov. 2007;6(3):231-48.

3. Gao P, Akrami A, Alvarez F, Hu J, Li L, Ma C, et al. Characterization and optimization of AMG 517 supersaturatable self-emulsifying drug delivery system (S-SEDDS) for improved oral absorption. J Pharm Sci. 2009;98(2):516-28.

4. Anby MU, Williams HD, McIntosh M, Benameur H, Edwards GA, Pouton CW, et al. Lipid digestion as a trigger for supersaturation: evaluation of the impact of supersaturation stabilization on the in vitro and in vivo performance of self-emulsifying drug delivery systems. Mol Pharm. 2012;9(7):2063-79.

5. Cuine JF, McEvoy CL, Charman WN, Pouton CW, Edwards GA, Benameur $\mathrm{H}$, et al. Evaluation of the impact of surfactant digestion on the bioavailability of danazol after oral administration of lipidic self-emulsifying formulations to dogs. J Pharm Sci. 2008;97(2):995-1012.

6. Arnold YE, Imanidis G, Kuentz M. Study of drug concentration effects on in vitro lipolysis kinetics in medium-chain triglycerides by considering oil viscosity and surface tension. Eur J Pharm Sci. 2011;44(3):351-8.

7. Sek L, Porter CJH, Charman WN. Characterisation and quantification of medium chain and long chain triglycerides and their in vitro digestion products, by HPTLC coupled with in situ densitometric analysis. J Pharmaceut Biomed. 2001;25(3-4):651-61.

8. Dressman J, Schamp K, Beltz K, Alsenz J. Characterizing release from lipid-based formulations. In: Hauss DJ, editor. Oral lipid-based formulations: enhancing the bioavailability of poorly water-soluble drugs. New York: Informa Healthcare; 2007.

9. Zangenberg NH, Mullertz A, Kristensen HG, Hovgaard L. A dynamic in vitro lipolysis model I. Controlling the rate of lipolysis by continuous addition of calcium. Eur J Pharm Sci. 2001;14(2):115-22.

10. Zangenberg NH, Mullertz A, Kristensen HG, Hovgaard L. A dynamic in vitro lipolysis model II: evaluation of the model. Eur J Pharm Sci. 2001;14(3):237-44.

11. Williams HD, Sassene P, Kleberg K, Bakala-N'Goma JC, Calderone $\mathrm{M}$, Jannin $\mathrm{V}$, et al. Toward the establishment of standardized in vitro tests for lipid-based formulations, part 1: method parameterization and comparison of in vitro digestion profiles across a range of representative formulations. J Pharm Sci. 2012;10(9):3360-80.

12. Williams HD, Anby MU, Sassene P, Kleberg K, Bakala N, Goma J-C, et al. Toward the establishment of standardized in vitro tests for lipidbased formulations. 2. The effects of bile salt concentration and drug loading on the performance of type I, II, IIIA, IIIB, and IV formulations during in vitro digestion. Mol Pharm. 2012;9(11):3286-300.

13. Larsen AT, Sassene P, Mullertz A. In vitro lipolysis models as a tool for the characterization of oral lipid and surfactant based drug delivery systems. Int J Pharm. 2011;417(1-2):245-55.

14. Vertzoni M, Markopoulos C, Symillides M, Goumas C, Imanidis G, Reppas C. Luminal lipid phases after administration of a triglyceride solution of danazol in the fed state and their contribution to the flux of Danazol across Caco-2 cell monolayers. Mol Pharm. 2012;9(5):1189-98.
15. Kossena GA, Charman WN, Boyd BJ, Porter CIH. Influence of the intermediate digestion phases of common formulation lipids on the absorption of a poorly water-soluble drug. J Pharm Sci. 2005;94(3):481-92.

16. Fatouros DG, Deen GR, Arleth L, Bergenstahl B, Nielsen FS, Pedersen JS, et al. Structural development of self nano emulsifying drug delivery systems (SNEDDS) during in vitro lipid digestion monitored by small-angle x-ray scattering. Pharm Res. 2007;24(10):184453.

17. Fatouros DG, Walrand I, Bergenstahl B, Mullertz A. Colloidal structures in media simulating intestinal fed state conditions with and without lipolysis products. Pharm Res. 2009;26(2):361-74.

18. Kleberg K, Jacobsen F, Fatouros DG, Mullertz A. Biorelevant media simulating fed state intestinal fluids: colloid phase characterization and impact on solubilization capacity. J Pharm Sci. 2010;99(8):352232.

19. Kossena GA, Boyd BJ, Porter CJH, Charman WN. Separation and characterization of the colloidal phases produced on digestion of common formulation lipids and assessment of their impact on the apparent solubility of selected poorly water-soluble drugs. J Pharm Sci. 2003;92(3):634-48.

20. Mohsin K, Long MA, Pouton CW. Design of lipid-based formulations for oral administration of poorly water-soluble drugs: precipitation of drug after dispersion of formulations in aqueous solution. J Pharm Sci. 2009;98(10):3582-95.

21. Patton JS, Carey MC. Watching fat digestion. Science. 1979;204(4389):145-8.

22. Bevernage J, Forier T, Brouwers J, Tack J, Annaert P, Augustijns P. Excipient-mediated supersaturation stabilization in human intestinal fluids. Mol Pharm. 2011;8(2):564-70.

23. Bevernage J, Brouwers J, Annaert P, Augustijns P. Drug precipitationpermeation interplay: Supersaturation in an absorptive environment. Eur J Pharm Biopharm. 2012;82(2):424-8.

24. Sassene PJ, Knopp MM, Hesselkilde JZ, Koradia V, Larsen A, Rades T, et al. Precipitation of a poorly soluble model drug during in vitro lipolysis: characterization and dissolution of the precipitate. J Pharm Sci. 2010;99(12):4982-91.

25. Warren DB, Anby MU, Hawley A, Boyd BJ. Real time evolution of liquid crystalline nanostructure during the digestion of formulation lipids using synchrotron small-angle x-ray scattering. Langmuir. 2011;27(15):9528-34.

26. Cornel J, Lindenberg C, Mazzotti M. Quantitative application of in situ ATR-FTIR and Raman spectroscopy in crystallization processes. Ind Eng Chem Res. 2008;47(14):4870-82.

27. Hu YR, Liang JK, Myerson AS, Taylor LS. Crystallization monitoring by Raman spectroscopy: simultaneous measurement of desupersaturation profile and polymorphic form in flufenamic acid systems. Ind Eng Chem Res. 2005;44(5):1233-40.

28. Caillet A, Puel F, Fevotte G. In-line monitoring of partial and overall solid concentration during solvent-mediated phase transition using Raman spectroscopy. Int J Pharm. 2006;307(2):201-8.

29. Ono T, ter Horst JH, Jansens PJ. Quantitative measurement of the polymorphic transformation of L-glutamic acid using in-situ Raman spectroscopy. Cryst Growth Des. 2004;4(3):465-9.

30. Scholl J, Bonalumi D, Vicum L, Mazzotti M, Muller M. In situ monitoring and modeling of the solvent-mediated polymorphic transformation of L-glutamic acid. Cryst Growth Des. 2006;6(4):881-91.

31. Pienack N, Bensch W. In-situ monitoring of the formation of crystalline solids. Angew Chem Int Ed. 2011;50(9):2014-34.

32. Arnold YE, Imanidis G, Kuentz MT. Advancing in-vitro drug precipitation testing: new process monitoring tools and a kinetic nucleation and growth model. J Pharm Pharmacol. 2011;63(3):333-41.

33. Reis MM, Araujo PHH, Sayer C, Giudici R. Spectroscopic on-line monitoring of reactions in dispersed medium: chemometric challenges. Anal Chim Acta. 2007;595(1-2):257-65. 
34. Fernandez S, Jannin V, Rodier JD, Ritter N, Mahler B, Carriere F. Comparative study on digestive lipase activities on the self emulsifying excipient Labrasol®, medium chain glycerides and PEG esters. BBA - Mol Gell Biol L. 2007;1771(5):633-40.

35. Fernandez S, Rodier JD, Ritter N, Mahler B, Demarne F, Carriere $\mathrm{F}$, et al. Lipolysis of the semi-solid self-emulsifying excipient Gelucire ${ }^{\circledR} 44 / 14$ by digestive lipases. BBA - Mol Cell Biol L. 2008;1781(8):367-75.

36. Garner CW. Boronic acid inhibitors of porcine pancreatic lipase. J Biol Chem. 1980;255(11):5064-8.

37. Juenemann D, Jantratid E, Wagner G, Reppas G, Vertzoni M, Dressman JB. Biorelevant in vitro dissolution testing of products containing micronized or nanosized fenofibrate with a view to predicting plasma profiles. Eur J Pharm Biopharm. 2011;77(2):257-64.

38. Geladi P, Macdougall D, Martens H. Linearization and scattercorrection for near-infrared reflectance spectra of meat. Appl Spectrosc. 1985;39(3):491-500.

39. Barnes RJ, Dhanoa MS, Lister SJ. Standard normal variate transformation and de-trending of near-infrared diffuse reflectance spectra. Appl Spectrosc. 1989;43(5):772-7.

40. Rinnan A, van den Berg F, Engelsen SB. Review of the most common pre-processing techniques for near-infrared spectra. Trac-Trends Anal Chem. 2009;28(10):1201-22.

41. Geladi P, Kowalski BR. Partial least-squares regression - a tutorial. Anal Chim Acta. 1986;185:1-17.

42. Stillhart C, Kuentz M. Comparison of high-resolution ultrasonic resonator technology and Raman spectroscopy as novel process analytical tools for drug quantification in self-emulsifying drug delivery systems. J Pharmaceut Biomed. 2012;59:29-37.

43. Dimo K. Nucleation: Basic theory and applications. Oxford: Butterworth-Heinemann; 2000.

44. Kashchiev D, van Rosmalen GM. Review: Nucleation in solutions revisited. Cryst Res Technol. 2003;38(7-8):555-74.

45. Mullin JW. Crystallization. 4th ed. ed. Oxford: ButterworthHeinemann; 2001.

46. Sek L, Porter CJH, Kaukonen AM, Charman WN. Evaluation of the in-vitro digestion profiles of long and medium chain glycerides and the phase behaviour of their lipolytic products. J Pharm Pharmacol. 2002;54(1):29-41.

47. Kuentz M. Lipid-based formulations for oral delivery of lipophilic drugs. Drug Discov Today Technol. 2012;9(2):e97-e104.

48. Dahan A, Hoffman A. Use of a dynamic in vitro lipolysis model to rationalize oral formulation development for poor water soluble drugs: Correlation with in viwo data and the relationship to intraenterocyte processes in rats. Pharm Res. 2006;23(9):2165-74.

49. Hu YR, Wikstrom H, Byrn SR, Taylor LS. Analysis of the effect of particle size on polymorphic quantitation by Raman spectroscopy. Appl Spectrosc. 2006;60(9):977-84.
50. Boetker J, Rades T, Rantanen J, Hawley A, Boyd BJ. Structural elucidation of rapid solution-mediated phase transitions in pharmaceutical solids using in situ synchrotron SAXS/WAXS. Mol Pharm. 2012;9(9):2787-91.

51. Thomas N, Holm R, Mullertz A, Rades T. In vitro and in vivo performance of novel supersaturated self-nanoemulsifying drug delivery systems (super-SNEDDS). J Control Release. 2012;160(1):25-32.

52. Cuine JF, Charman WN, Pouton CW, Edwards GA, Porter CJH. Increasing the proportional content of surfactant (Cremophor EL) relative to lipid in self-emulsifying lipid-based formulations of danazol reduces oral bioavailability in beagle dogs. Pharm Res. 2007;24(4):748-57.

53. Dahan A, Hoffman A. The effect of different lipid based formulations on the oral absorption of lipophilic drugs: The ability of in vitro lipolysis and consecutive ex viwo intestinal permeability data to predict in vivo bioavailability in rats. Eur J Pharm Biopharm. 2007;67(1):96-105.

54. Porter CJH, Kaukonen AM, Boyd BJ, Edwards GA, Charman WN. Susceptibility to lipase-mediated digestion reduces the oral bioavailability of danazol after administration as a medium-chain lipid-based microemulsion formulation. Pharm Res. 2004;21(8):1405-12.

55. Porter CJH, Kaukonen AM, Taillardat-Bertschinger A, Boyd BJ, O'Connor JM, Edwards GA, et al. Use of in vitro lipid digestion data to explain the in vivo performance of triglyceride-based oral lipid formulations of poorly watersoluble drugs: Studies with halofantrine. J Pharm Sci. 2004;93(5):1110-21.

56. Kleberg K, Jacobsen J, Mullertz A. Characterising the behaviour of poorly water soluble drugs in the intestine: application of biorelevant media for solubility, dissolution and transport studies. J Pharm Pharmacol. 2010;62(11):1656-68.

57. Anderson BD, Marra MT. Chemical and related factors controlling lipid solubility. Bulletin Technique Gattéfosse. 1999:11-9.

58. Borgstrom B, Brockman HL. Lipases. 1983.

59. Birner-Grunberger R, Scholze H, Faber K, Hermetter A. Identification of various lipolytic enzymes in crude porcine pancreatic lipase preparations using covalent fluorescent inhibitors. Biotechnol Bioeng. 2004;85(2):147-54.

60. Bakala N'Goma J-C, Amara S, Dridi K, Jannin V, Carrière F. Understanding the lipid-digestion process in the GI tract before designing lipid-based drug-delivery systems. Ther Deliv. 2012;3(1):105-24.

61. Wang CS, Kuksis A, Manganaro F. Studies on the substratespecificity of purified human-milk lipoprotein-lipase. Lipids. 1982;17(4):278-84.

62. Mattson FH, Volpenhein RA. Digestion and absorption of triglycerides. J Biol Chem. 1964;239(9):2772-77. 